\title{
Medienarbeit: Chance und Gefahr zugleich - Wie ticken die Medien?
}

Christian Müller

\author{
Der Ruf nach mehr Öffentlichkeitsarbeit und verstärkter Medienpräsenz ist im Be- \\ rufsstand der Tierärzte immer wieder hörbar. Allerdings ist das so eine Sache mit den \\ Medien. Sie sind Chance und Gefahr in einem. Der Beitrag zeigt, wo die Gefahren \\ lauern - und was zu tun ist, damit der Medienauftritt zur Chance wird.
}

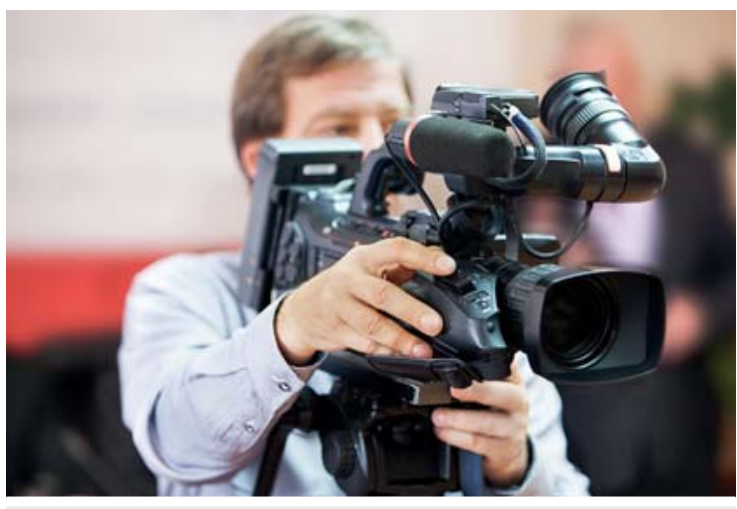

(c) Billion Photos.com - stock.adobe.com

Das Telefon klingelt, die Praxisassistentin nimmt den Anruf entgegen. Eine Journalistin des örtlichen Medienhauses ist am anderen Ende der Leitung und sagt, dass die Redaktion einen Beitrag rund um das Thema Tiermedizin in Arbeit habe. Sie bittet nun um ein paar kurze Einschätzungen eines Experten zu spezifischen Fachfragen.

Tierarzt Jakob Muster, Inhaber der Kleintierpraxis Bellenbach, lässt sich die Medienschaffende spontan durchstellen. Er kann sich ja mal anhören, was die Journalistin wissen möchte - und ihr, wenn möglich, weiterhelfen.

\section{Harmloses Porträt über „Fiffi-Omi“}

Die Journalistin erzählt, dass sie ein Porträt über eine betagte, alleinstehende Dame aus der Umgebung schreibe, die 3 Chihuahuas in ihrer Wohnung halte. Die Tiere seien ihr Ein und Alles, sie nehme sie überall hin mit, verfüttere ihnen nur qualitativ hochwertiges Fleisch aus der Metzgerei und ziehe ihnen im Winter purpurrote Wärmemäntelchen über. Mit ihrer auffälligen Zuneigung zu ihren winzigen Hunden habe sie sich im Ort den Übernamen „Fiffi-Omi“ eingehandelt.
Einer der Hunde sei schon seit längerem schwer krank und die Dame gebe für die medizinische Versorgung eigenen Angaben zufolge ein Vermögen aus. So werde dieser Tage der bereits 3. operative Eingriff am bedauernswerten kleinen Vierbeiner vorgenommen.

Was die Journalistin vom Experten nun wissen möchte, ist, ob so kostspielige Eingriffe bei Haustieren wirklich immer verhältnismäßig sind und ob in den letzten Jahren ein genereller Trend hin zur Vermenschlichung der Haustiere zu beobachten sei.

Mit ihren Fragen rennt die Journalistin bei Jakob Muster offene Türen ein. Er selber ist seit über 20 Jahren praktizierender Tierarzt und hat selber beobachtet, dass sich die Tiermedizin mehr und mehr an die Humanmedizin annähert. Er selber steht dieser Entwicklung kritisch gegenüber.

\section{Differenziert, offen und ehrlich}

So entwickelt sich ein angeregter Austausch am Telefon. Jakob Muster gibt an, dass sich die Bandbreite tiermedizinischer Eingriffe im Verlauf der letzten 2 Jahrzehnte erweitert hat und dass auch immer öfters größere Eingriffe bei Haustieren vorgenommen werden. Er berichtete davon, dass er vor Jahresfrist eine Chemotherapie für eine an Krebs erkrankte Katze durchgeführt hatte - und dies ohne realistische Aussicht auf Erfolg. Eigentlich habe er nur die Leidenszeit des Tieres verlängert, gibt Muster unumwunden zu. Natürlich machte er die Therapie auf eindringlichen Wunsch der Katzenbesitzerin. Letztlich entscheide der Klient, ob ein Eingriff gemacht wird oder nicht. Aber persönlich habe er schon seine Vorbehalte. Und das habe er der Klientin auch gesagt.

Die Journalistin hakt nach - und Muster antwortet frisch von der Leber: „Ja, mitunter handeln Tierbesitzer aus eigennützigen Motiven und tun ihren Tieren damit keinen Gefallen.“ Er teile diese Erfahrung mit verschiedenen 
Berufskollegen. „Diese falsche Tierliebe stimmt mich manchmal schon etwas nachdenklich."

Nach 15 Minuten, in denen viel gefragt und gesagt wird, bedankt sich die Journalistin für die wertvollen Inputs. Sie werde Dieses oder Jenes in ihren Beitrag einfließen lassen.

\section{Das böse Erwachen}

Zwei Stunden später, während des Mittagessens, erzählt Jakob Muster seiner Frau vom „Medientermin“. Sie reagiert verhalten. In Ihren Augen sind die gemachten Aussagen teilweise heikel, weil sie falsch interpretiert werden könnten. Sie gibt zu bedenken, dass insbesondere die Erwähnung der Katzen-Chemotherapie ungeschickt war. Die betroffene Klientin könnte das womöglich mitbekommen und würde das bestimmt nicht schätzen.

Jetzt, wo er seine Frau so hört, teilt er ihre Skepsis. Zurück in der Praxis, meldet er sich darum als Erstes in der Redaktion, um einzelne seiner Aussagen zu relativieren und vor allem um sicherzustellen, dass das Beispiel mit der Katzen-Chemo keine Erwähnung findet.

„Zu spät“, bekommt er zu hören. Der Beitrag sei seit rund 1 Stunde online geschaltet und seither schon zig Mal angeklickt worden. Jakob Muster fährt den Computer hoch und navigiert sich auf die Newsplattform des Medienhauses - um sich dann verwundert die Augen zu reiben. An prominenter Stelle findet sich ein Foto von Rosi Meier, der betagten, alleinstehenden Dame und ihren 3 geschniegelten Chihuahuas. Darunter die Schlagzeile: „Tierliebe und ihre absurden Auswüchse“. Der Beitrag ist emotional aufgeladen, beschreibt das Leben der einsamen Rosi Meier und die ungewöhnliche Betreuung, die sie ihren 3 Hündchen zuteilwerden lässt. In einer 2. Hauptrolle agiert Tierarzt Jakob Muster als vehementer Kritiker übertriebener Tierliebe. Er bildet mit seinen pointierten Aussagen gewissermaßen den Gegenpool zu Rosi Meier. Das Ganze liest sich wie ein Schlagabtausch. Auch das Beispiel mit der fragwürdigen Chemotherapie für die Katze wird im Beitrag beschrieben.

So hatte sich Jakob Muster das definitiv nicht vorgestellt. Seinem Empfinden nach sind seine differenzierten Argumentationen und Aussagen aus dem Kontext gerissen, reduziert und zugespitzt wiedergegeben worden. Er hatte es gut gemeint mit der Journalistin - und wird nun derart „in die Pfanne gehauen“. Er befürchtet einen Reputationsschaden für sich und seine Praxis. Sein Frust ist riesig.

\section{Medien sind Chance und Gefahr zugleich}

Das Beispiel mit Rosi Meier und Jakob Muster ist frei erfunden - aber ganz nah an der Realität gebaut. Solche
WAS WIR SAGEN, GEHÖRT UNS

Ob Deutschland, Österreich oder Schweiz: Es gilt „das Recht am eigenen Wort“. Will heißen: Was wir sagen, gehört uns.

Diese eindeutige Regelung der „Besitzverhältnisse“ ist in Bezug auf die Medienarbeit von Bedeutung. Daraus ableiten lässt sich nämlich ein rechtlicher Anspruch auf das Gegenlesen von gemachten Aussagen.

Konkret bedeutet das: Wer einer Zeitung, einer Zeitschrift oder einem Online-Portal gegenüber Auskunft gibt, darf seine Aussagen (dazu gehören alle direkten und indirekten Zitate; jedoch nicht der ganze Text) in einer angemessenen Zeitspanne vor der Publikation gegenlesen und autorisieren.

Die Einflussnahme auf diese Zitate ist allerdings eingeschränkt. Nicht erlaubt ist es, Aussagen hinzuzufügen, ins Gegenteil zu verkehren oder stark zu entkräften. Ebenso untersagt ist es, Fragen zu streichen, hinzuzufügen oder umzuformulieren.

Ist keine Einigung über den Wortlaut möglich, so bleibt der interviewgebenden Person als einzige Möglichkeit der Rückzug des gesamten Interviews respektive aller gemachten Aussagen. Das ist eine äußerst unpopuläre Maßnahme und wird sich letztlich auch kaum auszahlen, weil der Journalist den Vorgang mit großer Wahrscheinlichkeit transparent machen wird. Bei Fernseh- oder Videointerviews hat es sich eingebürgert, sich die Interview-Sequenzen direkt vor Ort an der Kamera vorspielen zu lassen. Aufnahmen können grundsätzlich beliebig oft wiederholt werden und gelten erst dann als autorisiert, wenn beide Parteien - also Interviewgeber und Journalist - damit einverstanden sind.

Sind die Aussagen für das Radio bestimmt, wird es kompliziert. Zwar besteht auch hier ein (theoretischer) Anspruch darauf, sich die ausgewählten Aussagen via Telefon vorspielen zu lassen. Diese Vorgehensweise entspricht jedoch nicht der gängigen Praxis, weil Radiostationen oft unter Zeitdruck produzieren.

Um Missverständnisse, Enttäuschungen, Hektik oder Konflikte zu vermeiden, empfiehlt es sich, die Erwartungen und Rahmenbedingungen rund um die Autorisierung der Aussagen im Vorfeld des Medientermins mit dem Journalisten klar abzusprechen.

oder ähnlich gelagerte Erfahrungen mit Medien passieren tagtäglich. Landauf, landab.

Die Schlussfolgerung, dass man deswegen Medienkontakte wenn immer möglich vermeiden sollte, wäre indes vorschnell und verfehlt. Umso mehr, als ja gerade im Berufsstand der Tierärzte die Forderung nach mehr Öffentlichkeitsarbeit immer mal wieder aufflammt. 
Tatsache ist: Medien sind Chance und Gefahr in einem. Die Kunst einer professionellen und wirkungsvollen Medienarbeit besteht darin, die Chancen zu nutzen und die Gefahren zu eliminieren oder zumindest klein zu halten. Darum ist es hilfreich, zu verstehen, wie die Medien ticken.

\section{Beachtung ist alles}

Der Strukturwandel von der gedruckten zur elektronischen Informationsverbreitung setzt den Medienmarkt einem harten Verdrängungskampf aus. Weltweit stehen die Verlagshäuser in einem erbitterten wirtschaftlichen Wettbewerb um Aufmerksamkeit und Werbeeinnahmen. Überleben kann nur, wer beachtet wird. Folglich geht es bei der Auswahl und Aufmachung von einzelnen Beiträgen in erster Linie darum, ein möglichst breites Publikum anzusprechen.

Generell hohe Beachtung bei den Medienkonsumenten finden Beiträge, die den Menschen und dessen Schicksal oder dessen Tun in den Fokus stellen. Personalisierung und Skandalisierung - und damit die Verbreitung von Emotionalität - prägen die heutige Medienarbeit. Soziale Netzwerke wie Facebook und Twitter begünstigen diese Entwicklung zusätzlich.

„Personalisierung“ meint, dass die Medien bestrebt sind, Themen - egal, aus welchem Bereich - wenn irgendwie möglich an Personen „aufzuhängen“. Dadurch bekommt das möglicherweise etwas trockene Sachthema ein Gesicht. Es wird lebendiger, greifbarer - und damit attraktiver. Bezogen auf das erfundene Fallbeispiel aus der Kleintierpraxis Bellenbach wird nun nachvollziehbar, weshalb nicht der allgemeine Trend der Annäherung von der Tieran die Humanmedizin im Fokus des Medienbeitrags steht, sondern die gegensätzlichen Haltungen der Protagonisten. Emotionalität zieht. Im Sinne einer höheren Beachtung wird der „Zweikampf“ zwischen Tierarzt Jakob Muster und Hundeliebhaberin Rosi Meier zelebriert.

"Skandalisierung“ wiederum bedeutet, dass ein Thema so ausgewählt und abgehandelt wird, dass sich eine möglichst breite Allgemeinheit über einen oder mehrere Aspekte moralisch entrüstet. Konflikte und Skandale sind für Medien dankbar, denn sie haben hohen Nachrichtenwert, will heißen, sie werden gut beachtet. Angesichts dessen liegt es auf der Hand, dass Medien bestimmte Themen und Vorgänge mitunter künstlich skandalisieren oder Konflikte zusätzlich anheizen und am Kochen lassen. Diesem Trend kann sich niemand entziehen.

\section{Nicht aus dem Sattel schießen}

Wie also hätte Tierarzt Jakob Muster auf die Medienanfrage reagieren sollen? Was hätte er besser machen können? Nachfolgend ein paar simple Tipps für den Umgang mit Medien.
Als Erstes ist festzuhalten: Medienanfragen gegenüber ist - den beschriebenen Gefahren zum Trotz - grundsätzlich wohlwollend zu begegnen. Denn wenn Sie als Fachperson zu einem allgemeinen Thema angefragt werden, bietet Ihr Auftritt die Chance, die eigene Kompetenz einer breiten Öffentlichkeit bekannt zu machen und damit Imagepflege für die eigene Institution zu betreiben. Und im Falle einer Krise, will heißen, wenn die Medienanfrage im Zusammenhang mit Vorwürfen oder Anschuldigungen Ihrer Institution oder Ihrer Person gegenüber erfolgt, ist es sogar ein absolutes Muss, den Medientermin wahrzunehmen. Auskunftsverweigerung ist in solchen Situationen eigentlich nie eine Option. Sie würde als Überforderung oder Schuldeingeständnis interpretiert und Gerüchten und Spekulationen Tür und Tor öffnen.

Hingegen ist dringend davon abzuraten, den Medien gegenüber aus dem Sattel heraus, also spontan und ohne jede Vorbereitung, Rede und Antwort zu stehen - selbst dann nicht, wenn Ihnen das Thema vertraut ist. Es ist legitim und entspricht der gängigen Praxis vom Journalisten zu erfahren, welche Stoßrichtung der geplante Beitrag haben soll und wer sonst noch im Beitrag zu Wort kommt. Antworten auf diese Fragen gibt es immer, denn Medienschaffende greifen ein Thema nicht einfach willkürlich auf, sondern sie verfolgen einen bestimmten Plan. Wenn Sie die genauen Sachverhalte kennen, können Sie sich ein klareres Bild von der potenziellen Berichterstattung machen, bestimmte Aspekte und Fragestellungen antizipieren und sich insgesamt besser vorbereiten.

Wichtig ist auch die Frage, in welcher Form, auf welchem Kanal und zu welchem Zeitpunkt die Publikation vorgesehen ist. Erscheint der Beitrag als Interview in der Tages-

\section{CHECKLISTE MEDIENTERMIN}

Bei Eingang der Anfrage sind folgende Fragen zu klären:

- Thema \& Stoßrichtung?

- Wer kommt alles zu Wort?

- Form, Kanal \& Publikationszeitpunkt?

- Autorisierung

Vor dem Medientermin sind folgende Vorbereitungen zu treffen:

- Fakten sammeln und plausibilisieren

- Haltung festlegen

- Kernbotschaften entwickeln und verinnerlichen

- Antworten auf heikle Fragen vorbereiten

Bei Auftritten vor der Kamera ist zu beachten:

- Aufnahmeort (mit-)bestimmen

- äußere Erscheinung auf Inhalt abstimmen

- nicht in die Kamera blicken

- authentisch bleiben 
zeitung von morgen? Oder bereits in 2 Stunden als Lauftext mit eingeflochtenen Statements auf der OnlinePlattform? Oder werden einzelne Aussagen im Radio gesendet?

Klarheit in diesen Fragen bildet die Grundlage für einen weiteren, zentralen Punkt der Vorabklärungen: die Autorisierung. Sie haben einen rechtlichen Anspruch darauf, zumindest Ihre eigenen, direkten oder indirekten Zitate gegenzulesen. Allerdings handelt es sich bei diesem Anspruch um eine Hol-Schuld. Viele Journalisten bieten diese Möglichkeit des Gegenlesens von sich aus an - aber längst nicht alle. Es ist also an Ihnen, einzufordern, dass Ihnen Ihre Zitate vorgelegt werden. Wenn Sie ein gutes Einvernehmen mit dem Journalisten haben, ist es gut möglich, dass er Ihnen den ganzen Text zur Voransicht zustellt. Wichtig ist im Prinzip nur, dass Sie die Rahmenbedingungen der Autorisierung genau klären.

\section{Kurz und bündig}

Wenn das geregelt ist, nehmen Sie sich Zeit, um sich zu überlegen, was Sie sagen möchten und was nicht. Plausibilisieren Sie die Ihnen bekannten Fakten und hüten Sie sich vor spekulativen Aussagen. Unrichtige Aussagen schaden der Glaubwürdigkeit - und sie lassen sich nur schwer korrigieren.
Legen Sie Ihre Haltung fest. Machen Sie dafür den Perspektivenwechsel. Versetzen Sie sich in die Empfänger Ihrer Aussagen und fragen Sie sich, wie Sie eine bestimmte Aussage formulieren müssen, damit sie richtig verstanden wird. Und machen Sie sich auch bewusst, wem Sie mit welcher Aussage möglicherweise auf die Füße treten könnten.

Verpacken Sie Ihre Haltung nun in kurze, verständliche Sätze - sogenannte Kernbotschaften. Eine Kernbotschaft bringt das Wesentliche in 15 bis 20 Sekunden Redezeit auf den Punkt. Gerade im komplexen, tiermedizinischen Bereich gilt es, die Dinge vereinfacht und für den Laien verständlich wiederzugeben. Ob mündlich oder schriftlich: Vermeiden Sie lange und differenzierte Ausführungen, Fachbegriffe oder Fremdwörter. Wenn es Ihnen nicht gelingt, die Sache verständlich rüberzubringen, dann macht das der Journalist für Sie. Er übersetzt, was er meint, gehört respektive gelesen zu haben. Und in diesem Moment haben Sie die Kontrolle über Ihre Aussagen selbstverschuldet aus der Hand gegeben.

Machen Sie sich weiter auch schon im Vorfeld des Medientermins bewusst, welche heiklen oder verfänglichen Fragestellungen in einem bestimmten Sachverhalt auf Sie zukommen könnten. Auch hier sollten Sie gewappnet 
sein und genau wissen, wie Sie reagieren, wenn die Frage tatsächlich auftaucht.

Hätte Tierarzt Jakob Muster diese einfache Herangehensweise beherzigt, hätte er ein paar wenige Minuten in die Vorbereitung investiert, hätte er bestimmt überlegtere Aussagen gemacht. Er wäre weder vom Publikationszeitpunkt noch von der Aufmachung des Beitrags überrascht worden.

\section{Korrespondenzadresse}

\section{Christian Müller}

Dimedio GmbH

Aathalstr. 34

8610 Uster

Schweiz

info@dimedio.ch

\section{Bibliografie}

DOI https://doi.org/10.1055/s-0044-100202

Kleintier konkret 2018; 21: 30-34

(c) Georg Thieme Verlag KG Stuttgart · New York ISSN 1434-9132

\section{MEDIENPROFIS}

Professioneller Umgang mit den Medien will geübt sein

Ein professioneller Umgang mit den Medien ist ein Schlüsselfaktor für den Erfolg. Die Anforderungen in diesem Bereich sind in den letzten Jahren allerdings drastisch gestiegen, denn die neuen Medien sorgen für eine Dynamisierung der Debatten.

Die Medienprofis der Dimedio GmbH beraten Führungskräfte aus Wirtschaft, Verwaltung und Politik im Umgang mit Medien. Wie ticken die Medien von heute? Wie lassen sich soziale Medien wirksam nutzen? Wie bringt man komplexe Zusammenhänge verständlich auf den Punkt? Und wie kommuniziert man mit den Medien im Krisenfall?

Unsere Kompetenzen:

- Medientraining

- Krisenkommunikation

- Kommunikationskonzepte

- Beratung im Umgang mit Medien

- Coaching in Medien- und Auftrittskompetenz

Dimedio $\mathrm{GmbH}$

Christian Müller \& Andreas Stutz

www.dimedio.ch 